\title{
RESEARCH NOTES \\ THE GENERALIZATION AND PROOF OF BERTRAND'S POSTULATE
}

\section{GEORGE GIORDANO}

Department of Mathematics

Physics and Computer Science

Rycrson Polytechnical Institute

Toronto, Ontario, Canada M5B 2K3

(Received May 21, 1985 and in revised form December 16, 1986)

ABSTRACT. The purpose of this paper is to show that for $0<r<1$ one can determine explicitly an $x_{0}$ such that $v$ $x \geq x_{0}, \exists$ at least one prime betwcen $x x$ and $x$. This is a generalization of Bertrand's Postulate. Furthermore, the same procedures are used 10 show that if one can find upper and lower bounds for $\theta(x)$ whose difference is $k x^{p}$, then $\exists$ a primc between $x$ and $x-K x^{p}$, where $k, K>0$ are constants, $0<\rho<1$ and $\theta(x)=\sum_{p \leq x} \ln p$, where $p$ runs over the primes.

KEY WORDS AND PHRASES. Bertrand's Postulate, Primes, Intervals, Explicit bound for one prime in an interval 1980 AMS SUBJECT CLASSIFICATION CODE. 10H15, $10 \mathrm{~J} 15$.

\section{INTRODUCTION.}

Several authors (for example [1], [2]) have discussed estimates for differences between consecutive primes. The result of this note is a consequence of [2], for example; however, the methods used here are elementary and give explicit bounds for the range of validity.

The proof uses the work done by Lowell Schoenfeld [3]. In fact it is based on Theorem $7^{\circ}$ from his paper which statcs that

$$
|\theta(x)-x|<0.0077629 x \ln x \quad \text { for } x \geq e^{2}
$$

where $\theta(x)=\sum_{p s i}$ Inp. (here $p$ runs over the primes). Furthermore, it is based on a simple idea: if $\theta(x)-\theta(r x)>0$ then there must be at least one prime between $\mathrm{rx}$ and $\mathrm{x}$ (here $0<\mathrm{r}<1$ ). The importance of Theorem 1 is the following. by setting $r=1 / 2$ we get Bertrand's Postulate. Hence, this theorem is a generalization of this postulate.

The importance of Theorem 2 is that it suggests that if $\rho=1 / 2$ then $\exists$ a prime between $x$ and $x-K x^{1 / n}$ wherc $K$ is a positive constant. Morcover, Theorems 18 and 19 of a paper by J. Barkley Rosser and Lowell Schoenfeld [4] give numerical evidence for the hypothesis of Theorem 2 in the case of $\rho=1 / 2$. Of course, if the Riemann hypothesis holds, then Theorem 10 of [3] states that

$$
|\theta(x)-x|<\frac{1}{8 \pi} \sqrt{x} \ln ^{2} x, \text { if } 599 \leq x .
$$

\section{THEOREMS, LEMMA AND THEIR PROOFS.}

THEOREM 1. Suppose $0<r<1$, let $a=1-r, b=(1-r) \ln r-.008(1+r)$ and $c=-.008 \ln r$. If $x>e^{22 / r}$ and $\ln x>\left(-b+\sqrt{b^{2}-4 a c}\right) / 2 a$, then $\exists$ prime $p$ s.t. $r x<p \leq x$.

PROOF. We have by definition $\theta(x)=\sum_{p \leq x} \ln p$. Given a certain $0<r<1$ we want to find an $x_{0}$ s.t. $v_{x}>x_{0} \exists a$ prime $p$ between $r x$ and $x$. This means $\theta(x)-\theta(r x)>0$. From (1.1) we have for $x \geq e^{n / r}$ the following:

$$
\theta(x)-\theta(r x)>x(1-.008 / \ln (x))-r x(1+.008 / \ln (r x)) .
$$

What we need is

$$
x(1-.008 / \ln (x))-r x(1+.008 / \ln (r x))>0
$$


After several manipulations this becomes

$$
(1-r) \ln ^{2} x+((1-r) \ln r-.008(1+r)) \ln x-.008 \ln r>0
$$

Let $y=\ln x$; then

$$
(1-r) y^{2}+((1-r) \ln r-.008(1+r)) y-.008 \ln r>0
$$

By letting $a=1-r, b=(1-r) \ln r-.008(1+r)$ and $c=-.008 \ln r$, we have

$$
a y^{2}+b y+c>0 \text {. }
$$

Therefore we must find $y_{0}$ such that $y_{y}>y_{0},(2.1)$ will hold. Let $z=a y^{2}+b y+c$; since $a>0$, the parabola opens upward.

By equating $z=0$ we have

$$
a y^{2}+b y+c=0
$$

Now consider all the different types of roots in (2.2). They are the following:

If (2.2) has complex roots then $\gamma y \in R,(2.1)$ will hold.

If (2.2) has a double real root then $\gamma y>-b / 2 a,(2.1)$ will hold

Finally if (2.2) has distinct real roots then $\gamma y>\left(-b+\sqrt{b^{2}-4 a c}\right) / 2 a,(2.1)$ will hold.

However, regardless of the type of roots (2.2) has, if $y>\left(-b+\sqrt{b^{2}-4 a c}\right) / 2 a$ then (2.1) will hold. But $y=\ln x$. Therefore, if $\ln x>\left(-b+\sqrt{b^{2}-4 a c}\right) / 2 a$ then $\exists$ is a prime in that interval. Q.E.D.

THEOREM 2. Suppose $0<p<1$, let $c, c^{\prime} \geq 0$ and $K>c^{\prime}+c$. If $x$ is sufficiently large and $x-c x^{\rho}<\theta(x)<x+c^{\prime} x^{\rho}$, then $\exists$ a prime p s.t. $x-K x^{\rho}<p<x$.

PROOF. We want to have a prime between $x-K x^{p}$ and $x$. This means that $\theta(x)-\theta\left(x-K x^{p}\right)>0$. From the hypothesis we have

$$
\theta(x)-\theta\left(x-K x^{p}\right)>x-c x^{p}-\left(x-K x^{p}+c^{\prime}\left(x-K x^{p}\right)^{p}\right) .
$$

What we nced is $x-c x^{p}-\left(x-K x^{p}+c^{\prime}\left(x-K x^{p}\right)^{p}\right)>0$. Again after manipulation we have $K>c+c^{\prime}\left(1-K / x^{1-p}\right)^{p}$, i.e. $\mathrm{K}>\mathrm{c}+\mathrm{c}^{\prime}$. Q.E.D.

LEMMA. Suppose $0<r<1$, let $a=1-r, b=(1-r) \ln r-.008(1+r)$ and $c=-.008 \ln r$. If $z=a y^{2}+b y+c$ and $r \rightarrow 1$, then $\left(-b / 2 a, c-b^{2} / 4 a\right) \rightarrow(+\infty,-\infty)$ (this point is the vertex of the parabola).

PROOF

$$
\begin{aligned}
z & =a y^{2}+b y+c \\
& =a(y+b / 2 a)^{2}+c-b^{2} / 4 a
\end{aligned}
$$

So

$$
\lim _{r \rightarrow 1} \mathrm{~b} / 2 \mathrm{a}=\lim _{\mathrm{r} \rightarrow 1}((1-\mathrm{r}) \ln r-.008(1+\mathrm{r})) /(1-\mathrm{r})=-\infty .
$$
$\lim _{r \rightarrow 1} c-b^{2} / 4 a=\lim _{r \rightarrow 1}-.008 \ln r-((1-r) \ln r-.008(1+r))^{2} /(4(1-r))=-\infty$. Q.E.D. The significance of this Lemma is that the closer $r$ is to 1 the larger $x$ has to be in order to have at least one prime between $r x$ and $x$. Of course, the lower bound for $x$ is given explicitly by Theorem 1 .

\section{FINAL COMMENTS}

It is obvious that with the aid of super computers we can find lower bounds for which Theorem 1 will still bc valid. Although $\mathrm{x}>\mathrm{e}^{22}$ is a relatively "large" number without the aid of a computer, one could use Theorem 8 in a paper of J. Barkley Rosser and Lowell Schoenfeld [5] to obtain similar results. However, not only is the co-efficient not as sharp as the one used in (1.1), but also for $r$ sufficiently close to 1 , the Lemma guarantees that $x$ becomes extremely large. In fact, by using Theorem 4 in [4] one can prove the following. Suppose $0<r<1$; let $a=2(1-r), b=2(1-r) \ln r-r-1$ and $c=-\ln r$. If $x>563 / r$ and $\ln x>\left(-b+\sqrt{b^{2}-4 a c}\right) / 2 a$, then $\exists$ prime p s.t. $\mathrm{rx}<\mathrm{p} \leq \mathrm{x}$.

Now a simple proof of Bertrand's Postulate can be found in any elementary number theory book, for example, Niven and Zuckerman [6]. Finally, improvements to the bounds of $\theta(x)$ will greatly increase the importance of Theorem 2. 


\section{AKNOWLEDGEMENTS:}

Originally this paper was based on the work done by J. Barkley Rosser and Lowell Schoenfeld [4]. However, the referee informed me that certain theorems had been improved, which I have now incorporated in this paper. I would like to thank him for his insight and also for other modifications.

Also, I am deeply indebted to Professor J. Repka for commenting and suggesting, changes on the style and content of the manuscript which were most helpful.

I would like to dedicate this paper to the memory of my teacher R.A. Smith.

\section{REFERENCES}

1. HEATH-BROWN, D.R. and GOLDSTON, D.A., "A Note on the Differences Between Consecutive Primes", Math Ann, 266 (1984), 317-320.

2. HEATH-BROWN, D.R. and IWANIEC, H. "On the Differences Between Consecutive Primes", Invent. Math. 55 (1979), 49-69.

3. SCHOENFELD, Lowell, "Sharper Bounds for the Chebyshev Functions $\theta(x)$ and $\psi(x)$. II", Math Comp. 30 (1976), 337-360.

4. ROSSER, J. Barkley and SCHOENFELD, Lowell, "Approximate Formulas for Some Functions of Prime Numbers", Illinois L Math. 6 (1962), 64-94.

5. ROSSER, J. Barkley and SCHOENFELD, Lowell, "Sharper Bounds for the Chebyshev Functions $\theta(x)$ and $\psi(x)$.", Math Comp. 29 (1975), 243-269.

6. NIVEN, Ivan and ZUCKERMAN, Herbert S., "An Introduction to the Theory of Numbers", Third Edition, John Wiley and Sons, New York, 1972. 


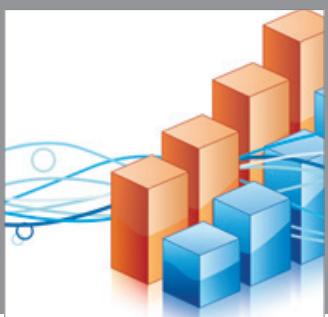

Advances in

Operations Research

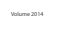

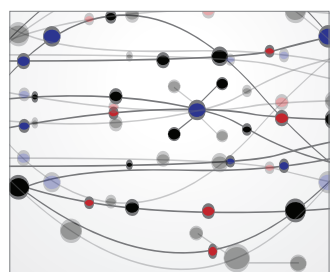

\section{The Scientific} World Journal
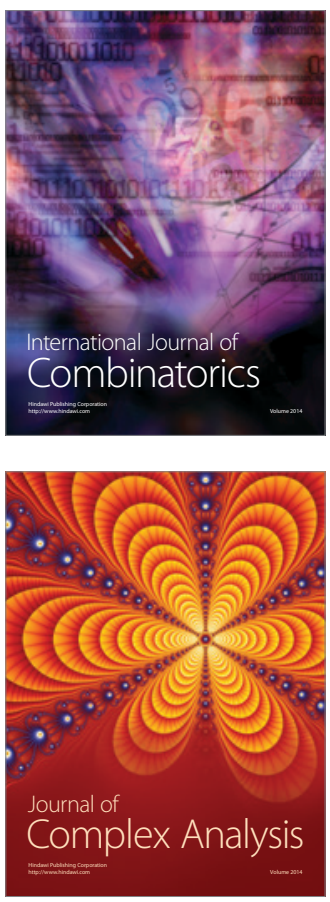

International Journal of

Mathematics and

Mathematical

Sciences
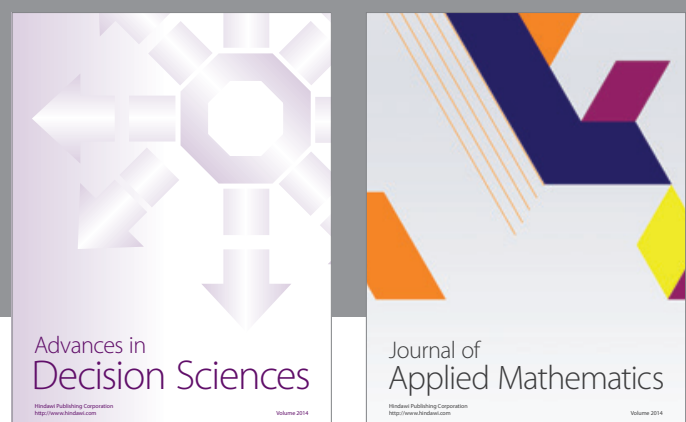

Journal of

Applied Mathematics
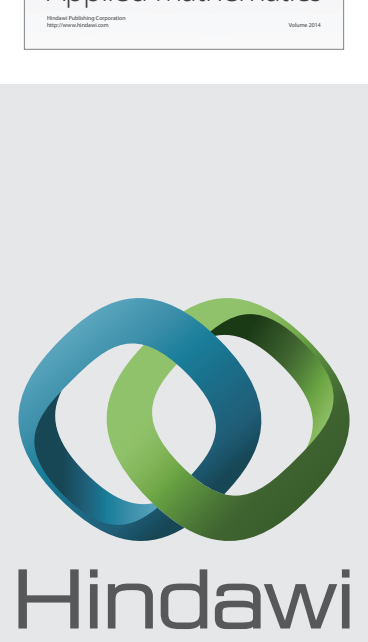

Submit your manuscripts at http://www.hindawi.com
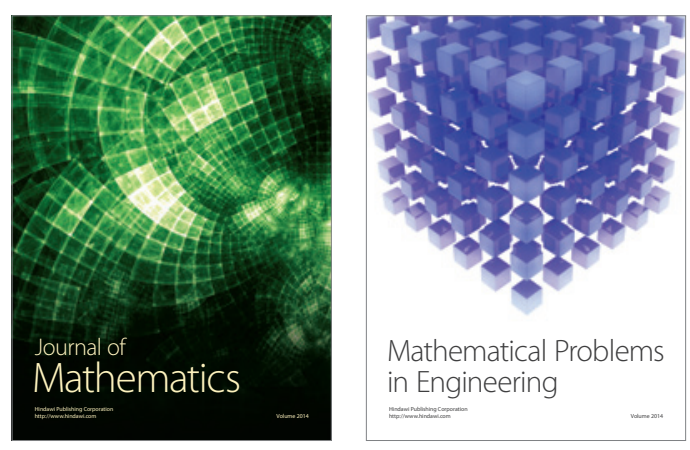

Mathematical Problems in Engineering
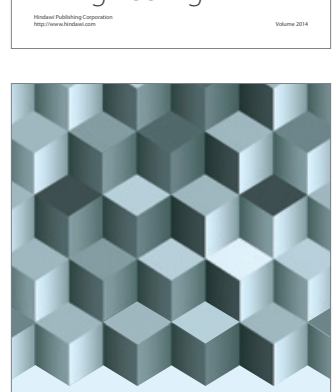

Journal of

Function Spaces
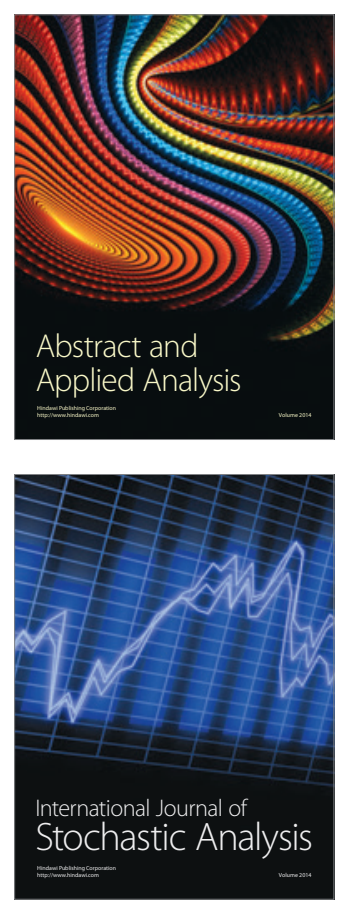

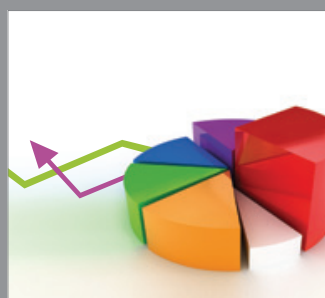

ournal of

Probability and Statistics

Promensencen
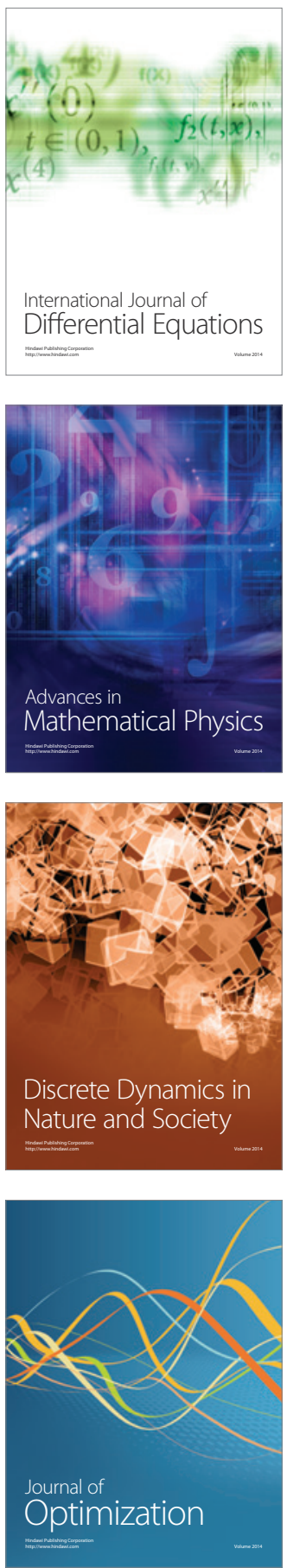